\title{
Rotation and gyration of finite two-dimensional modes
}

\author{
Kurt Bernardo Wolf ${ }^{1, *}$ and Tatiana Alieva ${ }^{2}$ \\ ${ }^{1}$ Instituto de Ciencias Físicas, Universidad Nacional Autónoma de México, Av. Universidad s/n, \\ Cuernavaca, Morelos 62251, Mexico \\ ${ }^{2}$ Universidad Complutense de Madrid, Facultad de Ciencias Físicas, Ciudad Universitaria s/n, Madrid 28040, Spain \\ *Corresponding author: bwolf@fis.unam.mx
}

Received October 2, 2007; accepted November 20, 2007; posted December 10, 2007 (Doc. ID 88150); published January 16, 2008

\begin{abstract}
Hermite-Gauss and Laguerre-Gauss modes of a continuous optical field in two dimensions can be obtained from each other through paraxial optical setups that produce rotations in (four-dimensional) phase space. These transformations build the SU(2) Fourier group that is represented by rigid rotations of the Poincaré sphere. In finite systems, where the emitters and the sensors are in $N \times N$ square pixellated arrays, one defines corresponding finite orthonormal and complete sets of two-dimensional Kravchuk modes. Through the importation of symmetry from the continuous case, the transformations of the Fourier group are applied on the finite modes. () 2008 Optical Society of America

OCIS codes: $070.2580,070.4560,080.2720,110.6980,120.4820,350.6980$.
\end{abstract}

\section{INTRODUCTION}

Paraxial optical systems with two-dimensional screens transform the four-dimensional optical phase space canonically, as specified by $4 \times 4$ matrices, which are symplectic in the geometric model, and through a corresponding integral transform in the scalar-wave model. Rotations of this manifold are performed with matrices that are simultaneously symplectic and orthogonal, forming the 4-parameter group of orthosymplectic matrices. This is a $4 \times 4$ representation of the two-dimensional unitary group $\mathrm{U}(2)=\mathrm{U}(1) \otimes \mathrm{SU}(2)$ [1] that we call the Fourier group. The matrices act on the coordinates $q_{k}$ and momenta $p_{k}, k \in\{x, y\}$, of classical phase space, and include the symmetric fractional Fourier transform (FT) in the central $\mathrm{U}(1)$ subgroup, antisymmetric FTs producing a fractional FT in the $x$ direction and its inverse FT in the $y$ direction, rotations in the $x y$ plane generated by an orbital angular momentum operator, and-the subject of our analysis-gyrators [2-4] that rotate jointly the phase planes $\left(q_{x}, p_{y}\right)$ and $\left(q_{y}, p_{x}\right)$. Hermite-Gauss beams are eigenfunctions of the symmetric and antisymmertic fractional FT, while Laguerre-Gauss beams are eigenfunctions of the symmetric FT and of rotations generated by the angular momentum operator [5].

In this paper we study the action of the $\mathrm{U}(2)$ Fourier group on finite systems, where the input and output signals consist of a finite number of complex data values arranged into $N \times N$ square pixellated images [6]. There, the 4-parameter Fourier group is represented by $N^{2} \times N^{2}$ matrices that are unitary and converge (weakly) to the corresponding integral kernels in the $N \rightarrow \infty$ limit [7]. The finite analogs of the Hermite-Gauss (HG) and LaguerreGauss (LG) modes will be presented in this paper and subjected to fractional gyration. To place these in proper context, we review the Fourier group and its distinguished modes in continuous systems in Section 2. The
SU(2) model for finite one- and two-dimensional systems is presented in Section 3 with the finite analog of HG modes: the two-dimensional Kravchuk modes. In Section 4 we import unitary rotation and gyration, thus defining the finite analog of the LG modes: Laguerre-Kravchuk modes. Both sets of modes, as well as their fractional gyrations - and generally their $\mathrm{U}(2)$ Fourier transformsare orthonormal and complete on the $N \times N$ points of the pixellated grid. Comments on extensions and applications are given in the concluding Section 5 .

\section{ROTATORS AND GYRATORS IN CONTINUOUS SYSTEMS}

Let $\bar{Q}_{x}, \bar{Q}_{y}, \bar{P}_{x}, \bar{P}_{y}$ be the well-known Schrödinger operators of position and momentum (indicating by the overbar that they refer to continuous models), whose generalized spectra are the real lines of their respective classical observables on phase space. Also, consider the (harmonic oscillator) number operators

$$
\bar{N}_{k}:=\frac{1}{2}\left(\bar{P}_{k}^{2}+\bar{Q}_{k}^{2}-1\right), \quad k \in\{x, y\},
$$

whose spectra in the Hilbert space $\mathcal{L}^{2}(\mathfrak{R})$ are the unbounded point sets $n_{k} \in\{0,1,2, \ldots\}$.

These Schrödinger operators satisfy the traditional Heisenberg and oscillator commutation relations with the unit operator 1 (and $\hbar=1$ ):

$$
\begin{gathered}
{\left[\bar{Q}_{k}, \bar{P}_{k^{\prime}}\right]=\mathrm{i} \delta_{k, k^{\prime}} 1, \quad\left[\bar{N}_{k}, \bar{Q}_{k^{\prime}}\right]=-\mathrm{i} \delta_{k, k^{\prime}} \bar{P}_{k},} \\
{\left[\bar{N}_{k}, \bar{P}_{k^{\prime}}\right]=\mathrm{i} \delta_{k, k^{\prime}} \bar{Q}_{k},}
\end{gathered}
$$

and $\left[\bar{Q}_{k}, \bar{Q}_{k^{\prime}}\right]=0,\left[\bar{P}_{k}, \bar{P}_{k^{\prime}}\right]=0$ as usual. Out of these operators one constructs the $u(2)$ algebra of generators of the $\mathrm{U}(2)$-FT subgroups as [8] 
symmetric FT $\bar{L}_{0}:=\frac{1}{4}\left(\bar{P}_{x}^{2}+\bar{P}_{y}^{2}+\bar{Q}_{x}^{2}+\bar{Q}_{y}^{2}-21\right)$

$$
=\frac{1}{2}\left(\bar{N}_{x}+\bar{N}_{y}\right) \text {, }
$$

antisymmetric FT $\bar{L}_{1}:=\frac{1}{4}\left(\bar{P}_{x}^{2}-\bar{P}_{y}^{2}+\bar{Q}_{x}^{2}-\bar{Q}_{y}^{2}\right)$

$$
=\frac{1}{2}\left(\bar{N}_{x}-\bar{N}_{y}\right) \text {, }
$$

$$
\text { gyration } \bar{L}_{2}:=\frac{1}{2}\left(\bar{P}_{x} \bar{P}_{y}+\bar{Q}_{x} \bar{Q}_{y}\right)
$$

$$
\text { rotation } \bar{L}_{3}:=\frac{1}{2}\left(\bar{Q}_{x} \bar{P}_{y}-\bar{Q}_{y} \bar{P}_{x}\right)=: \frac{1}{2} \bar{M}
$$

where $\bar{M}$ is the (physical) orbital angular momentum operator, whose eigenvalues should be integers. This Lie algebra is $u(2)$, characterized by its commutation relations

$$
\left[\bar{L}_{0}, \bar{L}_{k}\right]=0, \quad\left[\bar{L}_{i}, \bar{L}_{j}\right]=\mathrm{i} \bar{L}_{k}
$$

with $i, j, k$ a cyclic permutation of $1,2,3$. As is well known, $\mathrm{su}(2)$ also has a classical realization that generates rotations of the sphere, which is here the Poincaré 2-sphere shown in Fig. 1.

In the usual Schrödinger realization, the position operators $\bar{Q}_{x}, \bar{Q}_{y}$ are chosen to be diagonal, and the simultaneous eigenfunctions of $2 \bar{L}_{0}$ and $2 \bar{L}_{1}$ are the twodimensional HG functions $\mathrm{HG}_{n_{x}, n_{y}}^{(2)}(x, y)=\mathrm{HG}_{n_{x}}^{(1)}(x) \mathrm{HG}_{n_{y}}^{(1)}(y)$, with eigenvalues $n=n_{x}+n_{y} \in\{0,1,2, \ldots\}$ under $2 \bar{L}_{0}$ and $n_{x}-n_{y} \in\{-n,-n+2, \ldots, n\}$ under $2 \bar{L}_{1}$. On the other hand, the simultaneous eigenfunctions of $2 \bar{L}_{0}$ and $M=2 \bar{L}_{3}$ are the Laguerre-Gauss functions $\mathrm{LG}_{n, m}(x, y)$ with the principal quantum number $n$ and orbital angular momentum $m \in\{-n,-n+2, \ldots, n\}$; both are very well known [5]. The generator of gyration, $\bar{L}_{2}$ in Eq. (5), produces rotations of the Poincaré sphere [9] between the 1- and 3-axes of Fig. 1 ; for fixed $n$, gyrations will thus intertwine between the $n+1 \mathrm{HG}$ and the $n+1$ LG modes.

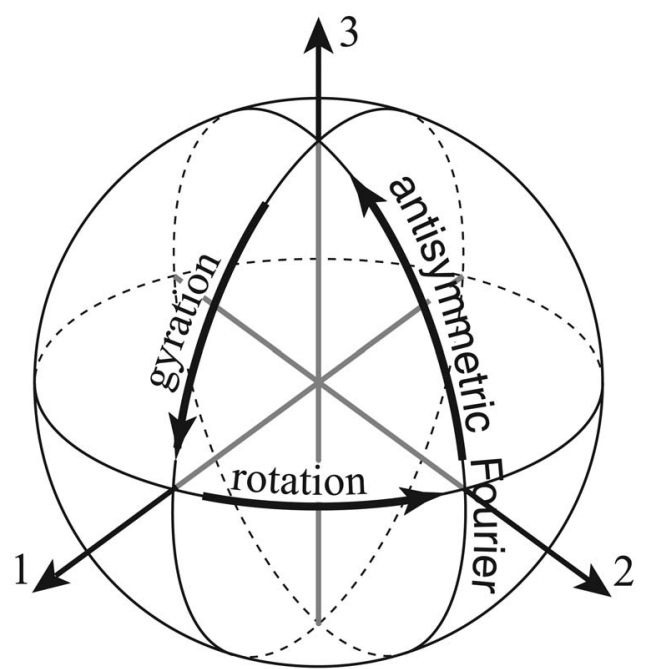

Fig. 1. Rotations of the Poincaré sphere generated by the su(2) algebra of operators $\bar{L}_{1}$ (antisymmetric Fourier), $\bar{L}_{2}$ (gyration), and $\bar{L}_{3}$ (rotation). As will be defined below, the rotation angles on the sphere are $2 \beta, 2 \gamma$, and $2 \alpha$, respectively.
The above operators, transformations, and wave functions of the continuous model have exact counterparts in the two-dimensional finite oscillator models, as developed in [10,11], where position space is an $N \times N$ square array of points, and where the separable HG modes are described by the product of two one-dimensional Kravchuk modes on a Cartesian grid, to be defined next.

\section{FINITE su(2) SYSTEMS}

In the one-dimensional finite model for optical systems we replace the Heisenberg and oscillator algebras in Eq. (2) by an su(2) algebra in the representation of dimension $N=2 j+1$, corresponding to spin $j$ (integer or half-integer), where it acts on $N$-component complex column vectors. Let $\left\{J_{i}\right\}_{i=1}^{3}$ stand for abstract generators of the su(2) algebra having the same commutation relations as $\left\{\bar{L}_{i}\right\}_{i=1}^{3}$ in Eq. (7), but carrying a distinct physical interpretation and role. The spectra of the operators $\left\{J_{i}\right\}_{i=1}^{3}$ are the observables in the su(2) model: position $Q=J_{1}$, momentum $P=J_{2}$, and number (or displaced energy) $N_{o}=J_{3}+j 1$-indicated without bars.

For the two dimensions of the screen, the following commutation relations are satisfied:

$$
\begin{gathered}
{\left[Q_{k}, P_{k^{\prime}}\right]=\mathrm{i} \delta_{k, k^{\prime}}\left(N_{k}-j 1\right), \quad\left[N_{k}, Q_{k^{\prime}}\right]=-\mathrm{i} \delta_{k, k^{\prime}} P_{k},} \\
{\left[N_{k}, P_{k^{\prime}}\right]=\mathrm{i} \delta_{k, k^{\prime}} Q_{k},}
\end{gathered}
$$

for $k \in\{x, y\}$, and all commutators with $k \neq k^{\prime}$ vanish. We note that the last two commutators in relations (8) are the geometric and dynamic oscillator Hamilton equations, the same as the last two in relations (2); only the first commutator, between positions and momenta, is different from the Heisenberg commutator of the Schrödinger harmonic oscillator model.

The six operators in relations (8) form a vector basis for the Lie algebra $\mathrm{su}(2)_{x} \oplus \mathrm{su}(2)_{y}$, and all have the spectrum $\{-j,-j+1, \ldots, j\}$. Positions, momenta, and numbers (of energy quanta) in the $x$ and $y$ directions will be thus intrinsically discrete and finite, consisting of $N=2 j+1$ equidistant points.

One-dimensional signals of $N$ complex data points are vectors in the Kronecker eigenbasis of the position operator $Q$, which is thus represented by a diagonal matrix. The finite harmonic oscillator functions are the overlaps between the eigenfunctions of $Q=J_{1}$ and those of the number operator $N_{o}=J_{3}$; they are given (as in quantum angular momentum theory) by a Wigner little- $d$ function $[12,13]$, for the angle $\frac{1}{2} \pi$ between them:

$$
\begin{aligned}
\Phi_{n}^{(N)}(q)=d_{n-j, q}^{j}\left(\frac{1}{2} \pi\right)=: & \frac{(-1)^{n}}{2^{j}} \sqrt{\left(\begin{array}{c}
2 j \\
n
\end{array}\right)\left(\begin{array}{c}
2 j \\
j+q
\end{array}\right)} K_{n}\left(j+q ; \frac{1}{2}, 2 j\right) \\
K_{n}\left(s ; \frac{1}{2}, 2 j\right) & ={ }_{2} F_{1}(-n,-j-s ;-2 j ; 2) \\
& =K_{s}\left(n ; \frac{1}{2}, 2 j\right),
\end{aligned}
$$

where $K_{n}\left(s ; \frac{1}{2}, 2 j\right)$ is a Kravchuk polynomial [14], and ${ }_{2} F_{1}(a, b ; c ; z)$ is the Gauss hypergeometric function. As written in Eq. (10), Kravchuk polynomials are orthogonal 
under summation over $s \in\{0,1, \ldots, 2 j\}$ with the binomial weight function $\left(\begin{array}{c}2 j \\ s\end{array}\right)$; the prefactor in relation (9) normalizes the $\Phi_{n}^{(N)}(q)$ under summation over $q \in\{-j,-j+1$, $\ldots, j\}$ with unit weight function, and so we call them Kravchuk functions. They are detailed in several papers $[10,11,15,16]$, where it is shown that they have all the desirable properties of the continuous HG modes, crucially including that when $N \rightarrow \infty,\left(\frac{1}{2} N\right)^{1 / 4} \Phi_{n}^{(N)}(x \sqrt{N / 2})$ converges uniformly to the $\mathrm{HG}_{n}(x)$ mode with $x \in \mathfrak{R}$ [7].

In two dimensions, $N \times N$ pixellated images are linear combinations of the simultaneous Kronecker eigenbases of $Q_{x}$ and $Q_{y}$, and correspondingly, the two-dimensional finite harmonic oscillator (Kravchuk) functions are the overlaps between the Kronecker eigenbasis of positions and the number eigenbasis of $N_{x}$ and $N_{y}$. They are simply given by [6]

$$
\begin{gathered}
\Phi_{n_{x}, n_{y}}^{(N)}\left(q_{x}, q_{y}\right):=\Phi_{n_{x}}^{(N)}\left(q_{x}\right) \Phi_{n_{y}}^{(N)}\left(q_{y}\right), \\
q_{x}, q_{y}, n_{x}-j, n_{y}-j \in\{-j,-j+1, \ldots, j\} .
\end{gathered}
$$

There are $N^{2}$ two-dimensional Kravchuk functions (11) that can be arranged by their quantum numbers $n_{x}$ and $n_{y}$ into the rhombus pattern shown in Fig. 2 .

\section{IMPORTATION OF SYMMETRY}

In the continuous model, modes arranged in the $\left(n_{x}, n_{y}\right)$ pattern of Fig. 2 form a tower with the same lower apex as the rhombus, but whose principal quantum number $n$ $=n_{x}+n_{y}$ can grow indefinitely. States with the same $n$ transform among themselves under the $U(2)$ Fourier group generated by the operators (3)-(6); it can thus be said that they form a multiplet of spin $\lambda=\frac{1}{2} n$, i.e., they behave as a vector of $2 \lambda+1$ components transforming under the corresponding matrix representation of the group of relations [8]. In the finite model however, $\mathrm{SU}(2)_{x}$ $\otimes S U(2)_{y}$ does not possess a subgroup to mix horizontally the modes of Fig. 2. Yet, one can import [17,18] these transformations using the same coefficients of the continuous model-as if they were the $n+1$ modes of the same multiplet. In [6] a gyration by $\frac{1}{2} \pi$ served to define "angular momentum" modes on a square $N \times N$ point array, which, under rotations by angles $\alpha$, multiply by the phases $e^{i m \alpha}$; they were also used to map square-to circular-pixellated screens in [19]. In this paper we identify the two-dimensional Kravchuk modes $\Phi_{n_{x}, n_{y}}^{(N)}\left(q_{x}, q_{y}\right)$ in relations (11) with the $\mathrm{HG}_{n}$ eigenmodes of $\bar{L}_{0}$ and $\bar{L}_{1}$, and we import both continuous rotations and gyrations.

\section{A. Rotations}

In the continuous model, a rotation by $\alpha$ is generated by the angular momentum operator $\bar{M}$ in relation (6) through $\overline{\mathcal{R}}(\alpha):=\exp (-\mathrm{i} \alpha \bar{M})$; because $\bar{M}=2 \bar{L}_{3}$, this corresponds to a rotation around the 3 -axis of the Poincaré sphere by the double angle $2 \alpha$. Since $\bar{L}_{3}$ commutes with $\bar{L}_{0}$, the eigenvalues of the latter, $\lambda=\frac{1}{2} n=\frac{1}{2}\left(n_{x}+n_{y}\right)$, are invariant under $\overline{\mathcal{R}}(\alpha)$, while the eigenfunctions of $\bar{L}_{1}$ will mix with linear combination coefficients that are the Wigner little- $d$ functions $d_{\mu, \mu^{\prime}}^{\lambda}(2 \alpha)$ [12]. These coefficients

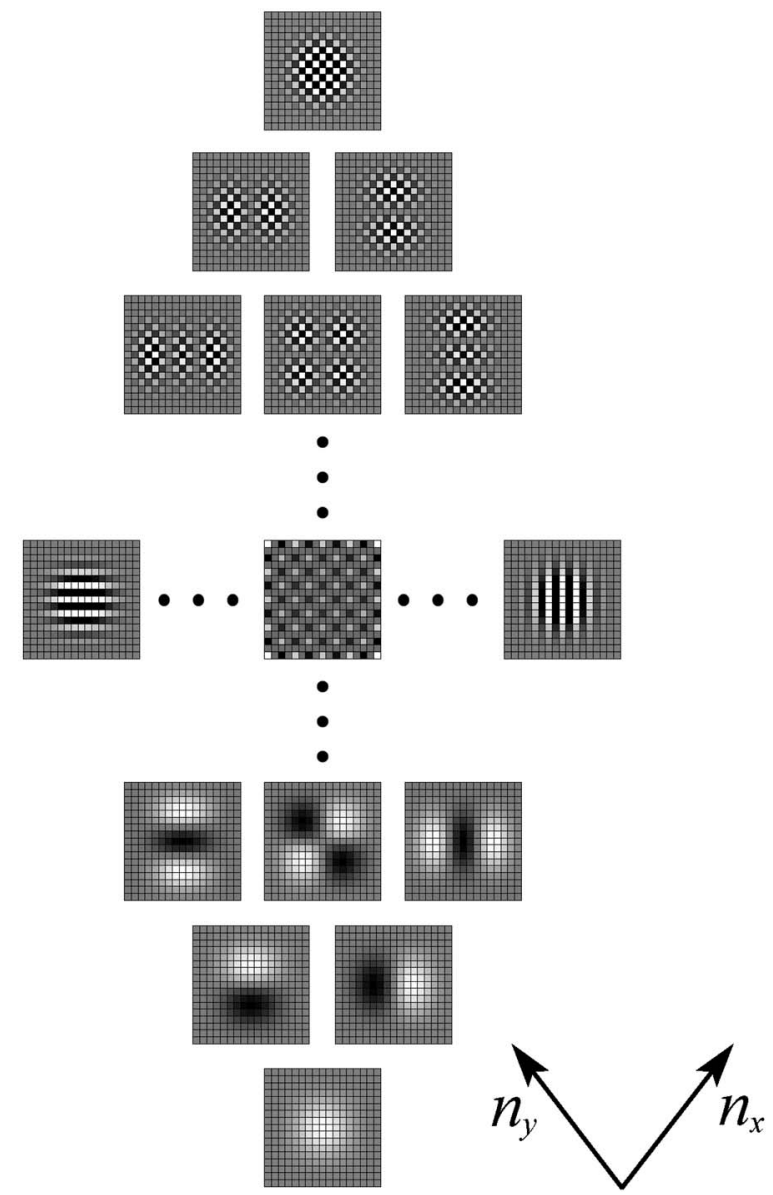

Fig. 2. Two-dimensional Kravchuk modes $\Phi_{n_{x}, n_{y}}^{(N)}\left(q_{x}, q_{y}\right)$ of the finite oscillator in a $17 \times 17$-point array $(j=8)$ classified by $n_{x}, n_{y}$ $\in\{0,1, \ldots, 16\}$. Each horizontal line of modes is characterized by the principal quantum number $n=n_{x}+n_{y}$. At each level $n$ in the lower triangle $0 \leqslant n \leqslant 2 j=N-1$ there are $n+1$ modes classified by $n_{x}-n_{y}$; in the upper triangle $2 j=N-1 \leqslant n \leqslant 4 j=2 N-2$ there are $4 j-n+1=2 N-n-1$ modes. The functions in the upper triangle of the rhombus equal their reflected partners in the lower triangle, but for a checkerboard of alternating signs.

are imported to the finite model so that the rotation of the two-dimensional Kravchuk modes is defined by

$$
\begin{aligned}
\mathcal{R}(\alpha) & : \Phi_{n_{x}, n_{y}}^{(N)}\left(q_{x}, q_{y}\right) \\
& :=\sum_{n_{x}^{\prime}+n_{y}^{\prime}=n} d_{1 / 2\left(n_{x}-n_{y}\right), 1 / 2\left(n_{x}^{\prime}-n_{y}^{\prime}\right)}^{n / 2}(2 \alpha) \Phi_{n_{x}^{\prime}, n_{y}^{\prime}}^{(N)}\left(q_{x}, q_{y}\right),
\end{aligned}
$$

where we note that the coefficients are real. The rotation (12) of the $n=4$ quintuplet ( $\operatorname{spin} \lambda=2$ ) of two-dimensional Kravchuk modes in Fig. 2 is shown in Fig. 3. This is a prime example of the importation of the symmetry from continuous to finite models.

\section{B. Antisymmetric FT}

In the continuous one-dimensional model revised in Section 2 , the number operator $\bar{N}$ generates the fractional FT group through $\mathcal{F}(\beta)=\exp (-\mathrm{i} \beta \bar{N})$, with $\beta$ counted modulo $2 \pi$; for $\beta=\frac{1}{2} \pi$ one has the traditional FT. Correspondingly, in the su(2) finite model (8), the domestic number operator $N_{o}$ generates the fractional Fourier-Kravchuk trans- 


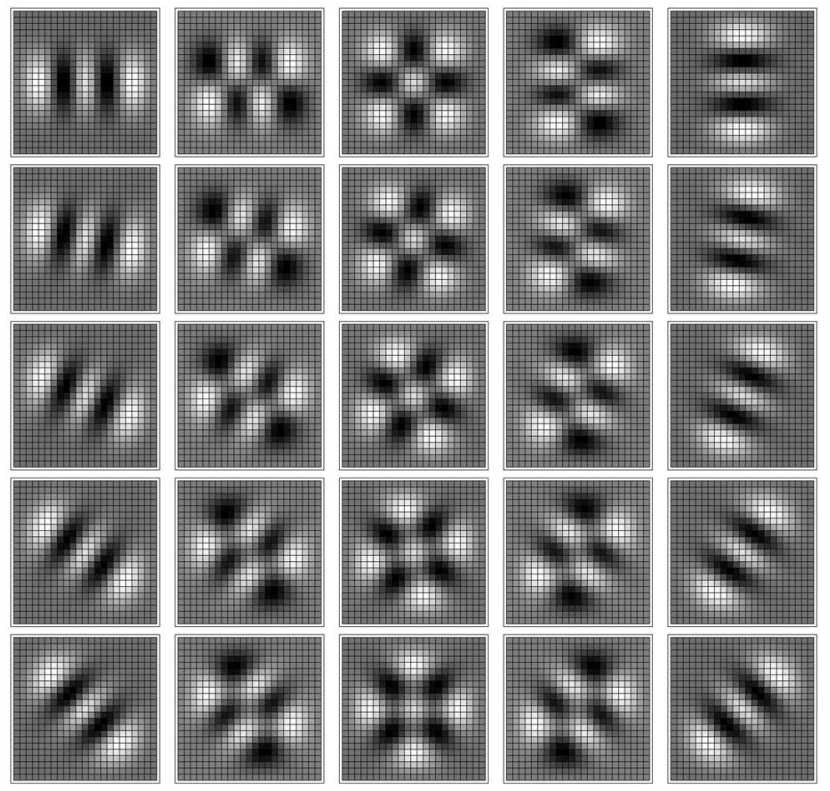

Fig. 3. Rotation of the five $n=4(\lambda=2)$ two-dimensional Kravchuk modes (top row), classified by $\frac{1}{2}\left(n_{x}-n_{y}\right) \in\{2,1,0,-1,-2\}$, in a $23 \times 23$ pixellated array $(N=23, j=11)$. Succesive rows show their rotations according to relation (12) by $\alpha=0, \pi / 16, \pi / 8$, $3 \pi / 16, \pi / 4$, corresponding to rotations of the Poincaré sphere by $2 \alpha$. The functions are real. Midgray pixels are 0 , black and white are minima and maxima, respectively.

form $\mathcal{K}(\beta)=\exp \left(-\mathrm{i} \beta N_{o}\right)$ [10], which is represented by unitary matrices of dimension $N=2 j+1$ forming a group with $\beta$ modulo $2 \pi$. When $N \rightarrow \infty$, the fractional FourierKravchuk transform becomes (weakly) the fractional Fourier integral transform [7].

Again, in the two-dimensional continuous model, the operator $\bar{L}_{1}=\frac{1}{2}\left(\bar{N}_{x}-\bar{N}_{y}\right)$ in relation (4) generates rotations of the Poincaré sphere around its 1 -axis. This is the antisymmetric fractional-FT: in the $x$ direction by the angle $\beta$ and in the $y$ direction by $-\beta$. In the finite case, the corresponding transformation is domestic: the antisymmetric fractional Fourier-Kravchuk transform $\mathcal{K}_{\mathrm{A}}(\beta)$ $:=\exp \left[-\mathrm{i} \beta\left(N_{x}-N_{y}\right)\right]$, which multiplies the twodimensional Kravchuk modes in relation (11) by phases,

$$
\mathcal{K}_{A}(\beta): \Phi_{n_{x}, n_{y}}^{(N)}\left(q_{x}, q_{y}\right)=\exp \left[-\mathrm{i} \beta\left(n_{x}-n_{y}\right)\right] \Phi_{n_{x}, n_{y}}^{(N)}\left(q_{x}, q_{y}\right) .
$$

On the Poincaré sphere, $\mathcal{K}_{\mathrm{A}}(\beta)=\exp \left(-2 \mathrm{i} \beta \bar{L}_{1}\right)$ is a rotation by $2 \beta$ around the 1 -axis. The symmetric fractional Fourier-Kravchuk transform corresponding to the symmetric fractional FT group $\mathrm{U}(1)$ generated by $\bar{L}_{0}=\frac{1}{2}\left(\bar{N}_{x}\right.$ $+\bar{N}_{y}$ ) in relation (3) will act on the two-dimensional Kravchuk modes as in Eq. (13), but with a phase $\exp (-\mathrm{i} \beta n)$.

\section{Gyrations}

Gyrations by $\gamma$ of the Poincaré sphere in the continuous model are generated by $\bar{L}_{2}$ in relation (5). These rotations around the 2-axis can be obtained from successive rotations of $\frac{1}{2} \pi$ around the 3-axis, $\gamma$ around the new 1-axis, and back by $-\frac{1}{2} \pi$ around the new 3 -axis. The first and third transformations are antisymmetric fractional FTs of angle $\frac{1}{4} \pi$,

$$
\mathcal{G}(\gamma):=\mathcal{F}_{A}\left(\frac{1}{4} \pi\right) \mathcal{R}(\gamma) \mathcal{F}_{A}\left(-\frac{1}{4} \pi\right) .
$$

We import this gyration to the finite model replacing $\mathcal{F}_{\mathrm{A}} \mapsto \mathcal{K}_{\mathrm{A}}$ in relations (13) and (14) - the latter is domestic to the finite model-and using the imported rotation of relation (12). Gyration thus acts on the two-dimensional Kravchuk modes $\Phi_{n_{x}, n_{y}}^{(N)}\left(q_{x}, q_{y}\right)$ with the same linear combination coefficients,

$$
\begin{aligned}
\Phi_{n_{x}, n_{y}}^{(N)}\left(q_{x}, q_{y} ; \gamma\right):= & \mathcal{G}(\gamma): \Phi_{n_{x}, n_{y}}^{(N)}\left(q_{x}, q_{y}\right) \\
= & \sum_{n_{x}^{\prime}+n_{y}^{\prime}=n} e^{-\mathrm{i} \pi\left(n_{x}-n_{y}\right) / 4} \\
& \times d_{1 / 2\left(n_{x}-n_{y}\right), 1 / 2\left(n_{x}^{\prime}-n_{y}^{\prime}\right)}^{n / 2}(2 \gamma) e^{+\mathrm{i} \pi\left(n_{x}^{\prime}-n_{y}^{\prime}\right) / 4} \\
& \times \Phi_{n_{x}^{\prime}, n_{y}^{\prime}}^{(N)}\left(q_{x}, q_{y}\right) .
\end{aligned}
$$

In Fig. 4 we show $n=4$ gyrated modes $\Phi_{n_{x}, n_{y}}^{(N)}\left(q_{x}, q_{y} ; \gamma\right)$ for various values of $0 \leqslant \gamma \leqslant \frac{1}{4} \pi$ corresponding to rotations over a quadrant of the Poincare sphere in Fig. 1. The upper row coincides with the two-dimensional Kravchuk modes because $d_{r, r^{\prime}}^{\lambda}(0)=\delta_{r, r^{\prime}}$, while the two bottom rows show the absolute value and phase of the modes that in [6] were called (finite) "angular momentum" modes; these could now be called Laguerre-Kravchuk modes
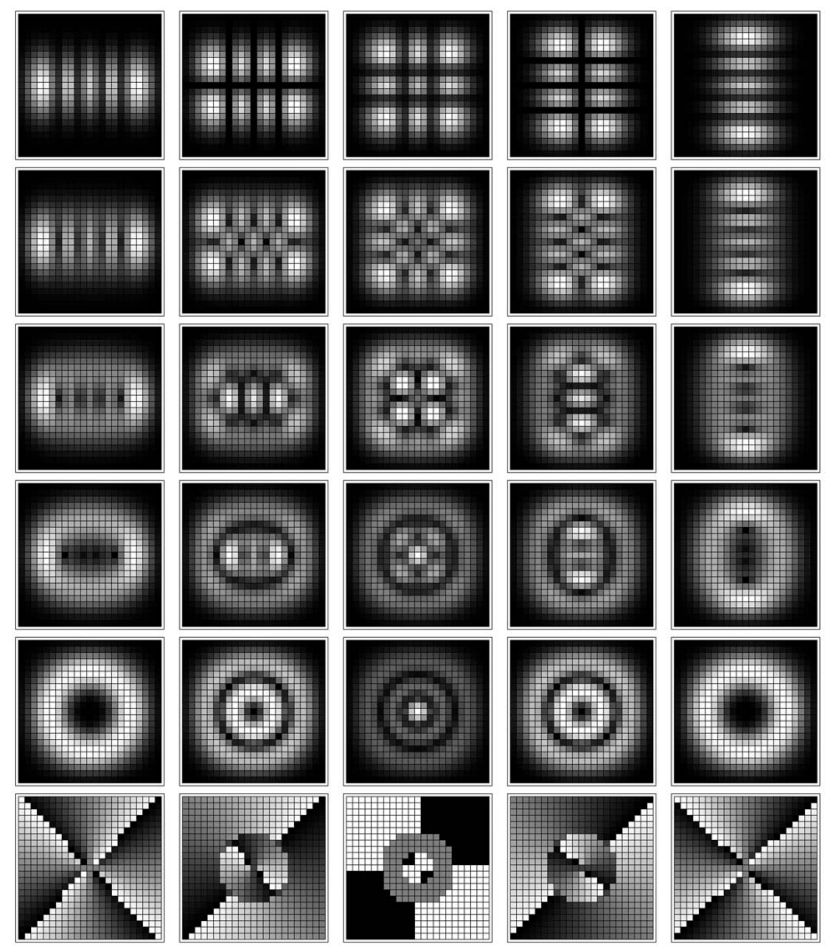

Fig. 4. Gyration according to relation (15) of the five $n=4(\lambda$ =2) two-dimensional Kravchuk modes, for angles $\gamma=0, \pi / 16$, $\pi / 8,3 \pi / 16, \pi / 4$. The top row corresponds to that in Fig. 3 but here shows absolute values (instead of real parts); the two bottom rows show the absolute values and phases of the $n+1$ Laguerre-Kravchuk modes classified by angular momentum $m$ $\in\{4,2,0,-2,-4\}$. Black is 0 , white are maxima; in the last row, the range from black to white is $(-\pi, \pi)$, except for the $m=0$ mode, which is real, but whose phase at $\pm \pi$ is numerically unstable. 
$\Lambda_{n, m}^{(N)}\left(q_{x}, q_{y}\right)$, characterized by the principal quantum number of the su(2) oscillator $n:=n_{x}+n_{y}=2 \lambda \in\{0,1, \ldots$, $2(N-1)\}$, and integer angular momentum $m \in\{-n$, $-n+2, \ldots, n\}$ :

$$
\begin{aligned}
\Lambda_{n, m}^{(N)}\left(q_{x}, q_{y}\right):= & e^{\mathrm{i} \pi m / 2} \Phi_{n_{x}, n_{y}}^{(N)}\left(q_{x}, q_{y} ; \frac{1}{4} \pi\right) \\
= & \sum_{n_{x}^{\prime}+n_{y}^{\prime}=n} e^{\mathrm{i} \pi\left(n_{x}^{\prime}-n_{y}^{\prime}\right) / 4} \\
& \times d_{\frac{1}{2} m, \frac{1}{2}\left(n_{x}^{\prime}-n_{y}^{\prime}\right)}^{n / 2}\left(\frac{1}{2} \pi\right) d_{n_{x}^{\prime \prime}-n / 2, q_{x}}^{n / 2}\left(\frac{1}{2} \pi\right) \\
& \times d_{n_{y}^{\prime}-n / 2, q_{y}}^{n / 2}\left(\frac{1}{2} \pi\right) .
\end{aligned}
$$

Under rotation and gyration, the modes can be arranged in the same rhombus pattern as the Kravchuk modes in Fig. 2, classified by the principal quantum number $n$ under $2 \bar{L}_{0}$, which indicates the row and is invariant. The columns were classified initially by their eigenvalues under $2 \bar{L}_{1}$, but under rotation (around the 3 -axis of the Poincaré sphere) or gyration (around its 2-axis), the classifying operator will rotate correspondingly to $2 \bar{L}_{2}$ or to $2 \bar{L}_{3}=\bar{M}$. Thus the $N^{2}$ Laguerre-Kravchuk modes $(n, m)$ are accomodated as shown in Fig. 5. They are of course also orthonormal and complete over the $N^{2}$ pixels $\left(q_{x}, q_{y}\right)$ of the screen, as are all modes obtained from the twodimensional Kravchuk modes $\Phi_{n_{x}}^{(N)} n_{y}\left(q_{x}, q_{y}\right)$ through transformations in the unitary $\mathrm{U}(2)$ Fourier group.

Last, it should be noted that the Wigner little- $d_{n-j, q}^{j}(\theta)$ functions in relation (9) are actually analytic functions of continuous position $-j-1<q<j+1$ in the $N=(2 j+1)$-point signal-with branchpoint zeros at $\pm(j+1)$. In the finite $\mathrm{su}(2)$ model there is thus an analytic continuation beyond the $N=(2 j+1)$ discrete points $q \in[-j, j]$ of the signal; this can be seen as an underlying continuum in the model, and extends to the two-dimensional functions considered here.

\section{CONCLUSIONS}

The Euler-angle parametrization of the SU(2) Fourier group elements that rotate the classical Poincaré sphere is

$$
\mathcal{D}(\psi, \theta, \phi)=\exp \left(-\mathrm{i} \psi \bar{L}_{3}\right) \exp \left(-\mathrm{i} \theta \bar{L}_{2}\right) \exp \left(-\mathrm{i} \phi \bar{L}_{3}\right) .
$$

We have seen that this group of transformations also acts unitarily on the functions of an $N \times N$ pixellated screen, because the subgroups of imported rotation and gyration, and the domestic Fourier-Kravchuk transforms mesh properly:

$$
\begin{aligned}
\mathcal{D}(\psi, \theta, \phi) & =\mathcal{K}_{A}\left(\frac{1}{2} \psi\right) \mathcal{G}\left(\frac{1}{2} \theta\right) \mathcal{K}_{A}\left(\frac{1}{2} \phi\right) \\
& =\mathcal{K}_{A}\left(\frac{1}{2} \psi+\frac{1}{4} \pi\right) \mathcal{R}\left(\frac{1}{2} \theta\right) \mathcal{K}_{A}\left(\frac{1}{2} \phi-\frac{1}{4} \pi\right) .
\end{aligned}
$$

The action of the Fourier group can be extended from the bases seen above to all $N \times N$ complex images on square pixellated screens.

Continuous optical systems (geometric and wave models) have a privileged group of linear canonical transformations, the 10-parameter real symplectic group $\operatorname{Sp}(4, \mathfrak{R})$

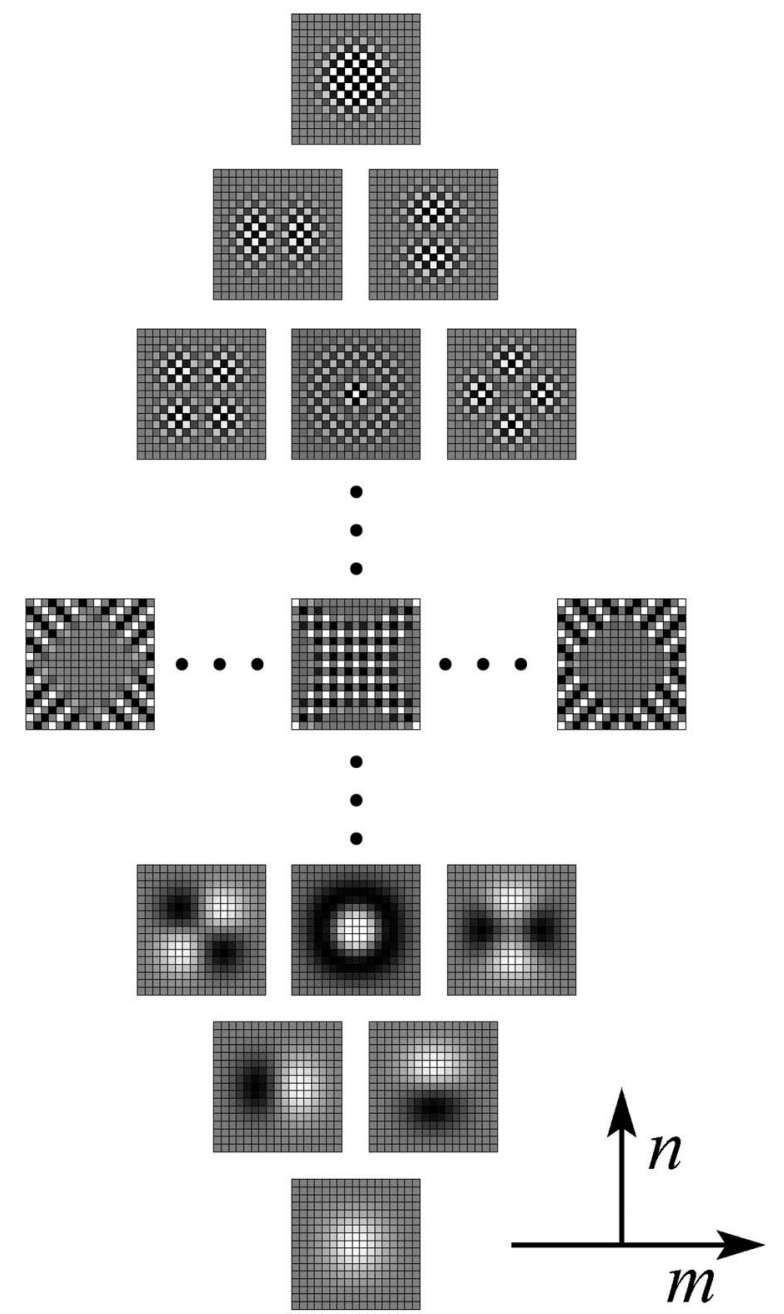

Fig. 5. Two-dimensional Laguerre-Kravchuk modes $\Lambda_{n, m}^{(N)}\left(q_{x}, q_{y}\right)$ for $N=2 j+1=17$ points $(j=8)$, classified by $n$ and $m$. Since $\left(\Lambda_{n, m}^{(N)}\right)^{*}=\Lambda_{n,-m}^{(N)}$, the real parts are shown for $m \geqslant 0$ and the imaginary parts for $m<0$. The highest angular momenta occur for $\pm m=2 j=n$ as if a quantum particle circulated around the periphery of the square; the $m=0$ modes have that particle moving along the diagonals.

for two-dimensional screens. This group contains the 4-parameter Fourier subgroup $U(2)$, which as we saw also applies to the finite model of optics with pixellated images. The remaining six parameters comprise generalized lenses and free flights, described by Fresnel transformations that shear the four-dimensional phase space, and image magnification with squeeze of momentum space. These have no linear counterpart in the finite model of optics. One-dimensional Fresnel and squeezing unitary transformations have been defined for the finite model as nonlinear transformations of the phase space sphere and understood as aberrations of the finite "paraxial" su(2) model $[20,21]$.

Finally we should remark that, since most optical sensor devices such as CCDs are finite and pixellated, and phase- and intensity-controlled object beams are often produced with similar liquid-crystal pixellated chips, it may be that experiments should be producing twodimensional Kravchuk and Laguerre-Kravchuk beams, 
rather than approximating their continuous, infinite counterparts by pointwise-sampled oscillator wave functions.

\section{ACKNOWLEDGMENTS}

T. Alieva acknowledges the Spanish Ministry of Education and Science for financial support (project TEC 200502180/MIC). K. B. Wolf acknowledges the support of the SEP-CONACYT (México) project IN102603 "Óptica Matemática." The authors are grateful to the UCM/ UNAM Collaboration Agreement for making this joint work possible. We appreciate Guillermo Krötzsch for assistance with the graphics, and Luis Edgar Vicent for Figs. 2 and 5.

\section{REFERENCES}

1. R. Simon and K. B. Wolf, "Fractional Fourier transforms in two dimensions," J. Opt. Soc. Am. A 17, 2368-2381 (2000).

2. M. J. Bastiaans and T. Alieva, "First-order optical systems with unimodular eigenvalues," J. Opt. Soc. Am. A 23, 1875-1883 (2006).

3. T. Alieva and M. J. Bastiaans, "Orthonormal mode sets for the two-dimensional fractional Fourier transform," Opt. Lett. 32, 1226-1228 (2007).

4. J. A. Rodrigo, T. Alieva, and M. L. Calvo, "Gyrator transform: properties and applications," Opt. Express 15, 2190-2203 (2007).

5. L. Allen, M. J. Padgett, and M. Babiker, "The orbital angular momentum of light" Progress in Optics, Vol. XXXIX, E. Wolf, ed. (Elsevier, 1999), pp. 294-374.

6. N. M. Atakishiyev, G. S. Pogosyan, L. E. Vicent, and K. B. Wolf, "Finite two-dimensional oscillator: I. The Cartesian model," J. Phys. A 34, 9381-9398 (2001).

7. N. M. Atakishiyev, G. S. Pogosyan, and K. B. Wolf, "Contraction of the finite one-dimensional oscillator," Int. J. Mod. Phys. A 18, 317-327 (2003).

8. A. Frank and P. Van Isacker, Algebraic Methods in
Molecular and Nuclear Structure Physics (Wiley, 1998); Sect. 1.1.

9. G. F. Calvo, "Wigner representation and geometric transformations of optical orbital angular momentum spatial modes," Opt. Lett. 30, 1207-1209 (2005).

10. N. M. Atakishiyev and K. B. Wolf, "Fractional Fourier-Kravchuk transform,” J. Opt. Soc. Am. A 14, 1467-1477 (1997).

11. N. M. Atakishiyev, G. S. Pogosyan, and K. B. Wolf, "Finite models of the oscillator," Phys. Part. Nucl. 36, Suppl. 3, 521-555 (2005).

12. L. C. Biedenharn and J. D. Louck, "Angular momentum in quantum mechanics," in Encyclopedia of Mathematics and Its Applications, Vol. 8, G.-C. Rota, ed. (Addison-Wesley, 1981), Sect. 3.6.

13. N. M. Atakishiyev and S. K. Suslov, "Difference analogs of the harmonic oscillator," Theor. Math. Phys. 85, 1055-1062 (1991).

14. M. Krawtchouk, "Sur une généralization des polinômes d'Hermite," C. R. Acad. Sci. Paris 189, 620-622 (1929).

15. N. M. Atakishiyev and K. B. Wolf, "Approximation on a finite set of points through Kravchuk functions," Rev. Mex. Fís. 40, 366-377 (1994)

16. N. M. Atakishiyev, L. M. Vicent, and K. B. Wolf, "Continuous vs. discrete fractional Fourier transforms," J. Comput. Appl. Math. 107, 73-95 (1999).

17. L. Barker, Ç. Çandan, T. Hakioğlu, and H. M. Ozaktas, "The discrete harmonic oscillator, Harper's equation, and the discrete fractional Fourier transform,” J. Phys. A 33, 2209-2222 (2000).

18. L. Barker, "Continuum quantum systems as limits of discrete quantum systems: II. State functions," J. Phys. A 34, 4673-4682 (2001).

19. L. E. Ruiz-Vicent, "Análisis de señales discretas finitas mediante el modelo de oscilador finito de su(2)," Ph. D. thesis (Universidad Autónoma del Estado de Morelos, Cuernavaca, 2007).

20. K. B. Wolf and G. Krötzsch, "Geometry and dynamics in the Fresnel transforms of discrete systems," J. Opt. Soc. Am. A 24, 2568-2577 (2007).

21. K. B. Wolf and G. Krötzsch, "Geometry and dynamics of squeezing in finite systems," J. Opt. Soc. Am. A 24, 2871-2878 (2007). 